\title{
To the Theory of Low-Dimensional Hydrogen Molecules
}

\author{
Vladimir V. Skobelev \\ Department of Physics, Moscow Polytechnic University, Moscow, Russia
}

Email address:

v. skobelev@inbox.ru

\section{To cite this article:}

Vladimir V. Skobelev. To the Theory of Low-Dimensional Hydrogen Molecules. American Journal of Physics and Applications.

Vol. 6, No. 5, 2018, pp. 115-127. doi: 10.11648/j.ajpa.20180605.12

Received: September 6, 2018; Accepted: October5, 2018; Published: November 9, 2018

\begin{abstract}
Using analytical and numerical methods, the possibility of existence of the elongated and flat hydrogen molecules $\mathrm{H}_{2}$ is first analyzed by analogy with the possibility of existence of low-dimensional single- and two-electron atoms previously proved theoretically (including us) and the impossibility of existence of the same multi-electron atom first pointed out in one of our previous works. In principle, conclusions of the present work can be verified experimentally, since low-dimensional, that is, one- and two-dimensional atoms were obtained experimentally long time ago. In our opinion, the material presented in the Appendix is of independent methodical interest because of its possible inclusion in traditional courses of quantum mechanics.
\end{abstract}

Keywords: One - Two-Dimensional Hydrogen Molecules, Binding Energy, Interaction Energy, Minimum

\section{Introduction}

Atoms with spatially one- and two-dimensional electronic structures were obtained experimentally long time ago, and existence of the same hydrogen-like atoms with analogous to three-dimensional characteristics was theoretically confirmed earlier also (including our previous works ) [1, 2, 3, 4]. However, it seems likely that as demonstrated in our work, multi-electron low-dimensional atoms do not exist [5]. In this regard, a natural question arises on the existence of onedimensional (that is, elongated) or two-dimensional (that is, flat) molecules comprising two identical hydrogen-like atoms ( $\mathrm{Ze}$ ) (at $Z=1$, this is molecular hydrogen $\mathrm{H}_{2}$ ).

This problem which was not touched at all in the literature known to us is the subject of the present work. References to other works having at least indirect relation to the present work in the above-mentioned aspect can be found in our works listed in the References. Presenting material below, we mainly focus on the conventional three-dimensional molecules $\mathrm{H}_{2}$ following the traditional course of quantum mechanics [6]. The equivalent more general interpretation is given in a classical book [7].

\section{Method}

Addition to the energy of three-dimensional hydrogen atoms due to their interaction considered as small perturbation is

$$
V_{S Y M T}(R)=\frac{K \pm A}{1 \pm S}
$$

for states symmetric and asymmetric with respect to permutations in the electron coordinates [6]. In this case, the functions $S \equiv S(R), K \equiv K(R)$, and $A \equiv A(R)$ of the distance $R \equiv|\vec{R}|$ between the nuclei are determined as follows:

$$
\begin{gathered}
S=\int d V \psi_{0}(\vec{r}) \psi_{0}(\vec{r}-\vec{R}) \\
K=\int d V \psi_{0}^{2}(\vec{r})\left[\frac{e^{2}}{R}-\frac{e^{2}}{|\vec{r}-\vec{R}|}\right] \\
A=\int d V \psi_{0}(\vec{r}) \psi_{0}(\vec{r}-\vec{R})\left[\frac{e^{2}}{R}-\frac{e^{2}}{|\vec{r}|}\right]
\end{gathered}
$$

where $K$ is the average energy of the Coulomb interaction of the hydrogen atom with the ion of the hydrogen atom [6]. $A$ - quantity has no classical analog and is the so-called exchange energy, and $\psi_{0}$ is the real wave function of the ground electron state in the hydrogen-like atom $(Z e)$.

Our purpose below is refinement of formulas (2-4) for the one-dimensional and two-dimensional molecules with the 
subsequent elucidation of the form of the dependence of functions (1) on the distance between the nuclei to elucidate the presence of a minimum of these functions in the negative region of their values at a certain distance corresponding to the equilibrium distance between the nuclei in the stable molecule comprising two hydrogen atoms.

First we note that generalization of these formulas to the spaces with dimensions $D=1,2$ considered as the subspaces of the three-dimensional space $D=3$ (and also with the real wave function of the ground state - see Eqs. (14) and (38) below) has the form

$$
\begin{gathered}
S=\int d \Gamma_{D} \psi_{0}(\vec{r}) \psi_{0}(\vec{r}-\vec{R}) \\
K=\int d \Gamma_{D} \psi_{0}^{2}(\vec{r})\left[\frac{e^{2}}{R}-\frac{e^{2}}{|\vec{r}-\vec{R}|}\right] \\
A=\int d \Gamma_{D} \psi_{0}(\vec{r}) \psi_{0}(\vec{r}-\vec{R})\left[\frac{e^{2}}{R}-\frac{e^{2}}{|\vec{r}|}\right]
\end{gathered}
$$

where $d \Gamma_{2} \equiv d S=d x d y, d \Gamma_{1}=d z$, including the case of $D=3$ also with $d \Gamma_{3} \equiv d V=d x d y d z$.

Let us now proceed in these formulas to dimensionless in a conventional sense variables and functions

$$
d \tilde{\Gamma}_{D}=\frac{d \Gamma_{D}}{\left(a_{Z}\right)^{D}}, \overrightarrow{\tilde{r}}=\frac{\vec{r}}{a_{Z}}, \overrightarrow{\tilde{R}}=\frac{\vec{R}}{a_{Z}}, \tilde{\psi}_{0}=\left(a_{Z}\right)^{D / 2} \psi_{0}
$$

with the normalization condition

$$
\int d \tilde{\Gamma}_{D} \tilde{\psi}_{0}^{2}(\overrightarrow{\tilde{r}})=1
$$

and designation

$$
a_{Z}=\frac{\hbar^{2}}{m_{e}\left(Z e^{2}\right)}
$$

Then Eqs. (5), (6) and (7) can be written in the form more convenient for the subsequent applications:

$$
S=\int d \tilde{\Gamma}_{D} \tilde{\psi}_{0}(\overrightarrow{\tilde{r}}) \tilde{\psi}_{0}(\overrightarrow{\tilde{r}}-\overrightarrow{\tilde{R}})
$$

$$
\begin{gathered}
K=\alpha(Z \alpha) m_{e} c^{2} \int d \tilde{\Gamma}_{D} \tilde{\psi}_{0}^{2}(\overrightarrow{\tilde{r}})\left[\frac{1}{\tilde{R}}-\frac{1}{|\overrightarrow{\tilde{r}}-\overrightarrow{\tilde{R}}|}\right] \\
A=\alpha(Z \alpha) m_{e} c^{2} \int d \tilde{\Gamma}_{D} \tilde{\psi}_{0}(\overrightarrow{\tilde{r}}) \tilde{\psi}_{0}(\overrightarrow{\tilde{r}}-\overrightarrow{\tilde{R}})\left[\frac{1}{\tilde{R}}-\frac{1}{|\overrightarrow{\tilde{r}}|}\right] \\
\alpha=\frac{e^{2}}{\hbar c} \approx \frac{1}{137}
\end{gathered}
$$

The parameter $a_{Z}$ given by Eq. (10) which at $Z=1$ is simply the Bohr radius enters conventionally into the normalization coefficient of dimensional wave function $\psi_{0}$. The dimensionless charge of the nucleus $Z$, designated by $Z^{\prime}$ in the variation method used in Section 5 to calculate the energy of the hydrogen molecule comprised in $a_{Z}$ as the variation parameter $[8,9][6,7]$.

In Section 3 below based on general formulas (11-13) we calculate analytically the corresponding functions $S, K, A$ depending on the dimensionless distance between the nuclei $\tilde{R} \rightarrow \tilde{L}=L / a_{Z}$ in the one-dimensional hydrogen molecule $\mathrm{H}_{2}$ with illustrative graphic representation of the interaction energy as a function of $\tilde{L}$ designating in this case the conventional distance $R$ by symbol $L$.

In Section 4, the same program is realized for the twodimensional molecule with designation of the dimensionless distance between the nuclei $\tilde{R}$ by analogy with the designation $R$ of the distance in the three-dimensional variant [6].

In Section 5, the variation method is used to calculate the energy of the two-dimensional and one-dimensional molecules using formulas presented in the Appendix which is also of independent (mainly methodical) interest. In Section 6 we discuss the results obtained.

\section{One-Dimensional Molecule. Derivation of General Relations by Analytical Methods}

The dimensionless in a conventional sense wave function of the one-dimensional hydrogen-like atom elongated along the $z$ - axis with the origin of coordinates in one of the nucleus has the form $[7,10])$ :

$$
\tilde{\psi}_{0} \equiv \tilde{\psi}_{0}(\varsigma), \tilde{\psi}_{0}(\varsigma)=\sqrt{2}|\varsigma| e^{-|\varsigma|}\left\{\begin{array}{l}
1, \varsigma>0 \\
P, \varsigma<0
\end{array}, \varsigma=\frac{z}{a_{Z}}\right.
$$

(traditionally we designate here the dimensionless in a conventional sense variable by the symbol $\varsigma$ and by $P= \pm 1$-the parity of the state). The energy

$$
E_{n}=-\frac{(Z \alpha)^{2}}{2 n^{2}} m_{e} c^{2}
$$

coincides with the three-dimensional case. 
Proceeding to calculation of the functions $S, K, A$ in Eqs. (1) and (11-13) in their prolongation to the one-dimensional space $D=1$ (see also Eq. (26-29) below) as a subspace of the three-dimensional space, we note that

Formula (11) in this one-dimensional case assumes the form

$$
S \equiv S\left(Z_{N}\right)=\int_{-\infty}^{\infty} d \varsigma \tilde{\psi}_{0}(\varsigma) \tilde{\psi}_{0}\left(\varsigma-\tilde{Z}_{N}\right)
$$

where $Z_{N}$ is the coordinate of one nucleus relative to another and $\tilde{Z}_{N}=Z_{N} / a_{Z}$.

Having designated the distance between the nuclei $L=\left|Z_{N}\right|$ and having introduced the dimensionless distance $\tilde{L}=\left|\tilde{Z}_{N}\right|$, this expression for $\tilde{Z}_{N} \equiv \tilde{L}>0$ with allowance for the parity of the states can be reduced to the form

$$
\begin{aligned}
S\left(\tilde{Z}_{N}>0\right) & =2\left[P P^{\prime} e^{-\tilde{L}} \int_{-\infty}^{0} d \varsigma|\varsigma|(\tilde{L}-\varsigma) e^{-|\zeta|+\varsigma}+e^{-\tilde{L}} P^{\prime} \int_{0}^{\tilde{L}} d \varsigma \varsigma(\tilde{L}-\varsigma) e^{-|\varsigma|+\varsigma}+e^{\tilde{L}} \int_{\tilde{L}}^{\infty} d \varsigma \varsigma \times(\varsigma-\tilde{L}) e^{-|\varsigma|-\varsigma}\right] \\
& =2\left[P P^{\prime} e^{-\tilde{L}} \int_{0}^{\infty} d \varsigma \varsigma(\tilde{L}+\varsigma) e^{-2 \varsigma}+e^{-\tilde{L}} P^{\prime} \int_{0}^{\tilde{L}} d \varsigma \varsigma(\tilde{L}-\varsigma)+e^{\tilde{L}} \int_{\tilde{L}}^{\infty} d \varsigma \varsigma(\varsigma-\tilde{L}) e^{-2 \varsigma}\right]
\end{aligned}
$$

Elementary calculation yields

$$
S\left(\tilde{Z}_{N}>0\right)=e^{-\tilde{L}}\left[\left(P P^{\prime}+1\right) \frac{1}{2}(\tilde{L}+1)+P^{\prime} \frac{1}{3} \tilde{L}^{3}\right] \equiv S(\tilde{L})
$$

For $\tilde{Z}_{N} \equiv-\tilde{L}<0$, we analogously obtain

$$
\begin{aligned}
S\left(\tilde{Z}_{N}<0\right) & =2\left[P P^{\prime} e^{\tilde{L}} \int_{\tilde{L}}^{\infty} d \varsigma \varsigma(\varsigma-\tilde{L}) e^{-|\varsigma|-\varsigma}+P e^{-\tilde{L}} \int_{-\tilde{L}}^{0} d \varsigma|\varsigma|(\varsigma+\tilde{L}) e^{-|\varsigma|-\varsigma}+e^{-\tilde{L}} \int_{0}^{\infty} d \varsigma \varsigma \times(\varsigma+\tilde{L}) e^{-|\varsigma|-\varsigma}\right] \\
& =2\left[P P^{\prime} e^{\tilde{L}} \int_{\tilde{L}}^{\infty} d \varsigma \varsigma(\varsigma-\tilde{L}) e^{-2 \varsigma}+e^{-\tilde{L}} P \int_{0}^{\tilde{L}} d \varsigma \varsigma(\tilde{L}-\varsigma)+e^{-\tilde{L}} \int_{0}^{\infty} d \varsigma \varsigma(\varsigma+\tilde{L}) e^{-2 \varsigma}\right]
\end{aligned}
$$

As can be seen from (16),(18),

$$
S\left(\tilde{Z}_{N}<0\right)=P P^{\prime} S\left(\tilde{Z}_{N}>0\right)
$$

Of course, since the function $S$ by analogy with the interaction energy given by Eq. (1) must be independent of the sign of $\tilde{Z}_{N}$ and must depend only on $\tilde{L}$, we obtain $P P^{\prime}=1$, that is, in any case the parities of electron states in the one-dimensional molecule $H_{2}$ should be identical: $P=P^{\prime}= \pm 1$.

Thus, with allowance for Eqs. (17) and (19), we obtain

$$
S(\tilde{L}) \rightarrow S^{( \pm)}(\tilde{L})=e^{-\tilde{L}}\left(1+\tilde{L} \pm \frac{1}{3} \tilde{L}^{3}\right)
$$

Expression (12) for $K$ after some transformations for $\tilde{Z}_{N}(\equiv \tilde{L})>0$ is written in the one-dimensional case in the form

$$
\begin{gathered}
K\left(Z_{N}>0\right)=\alpha(Z \alpha) m_{e} c^{2}\left\{\frac{1}{\tilde{L}}-2\left[\int_{0}^{\infty} d \varsigma \varsigma^{2} \frac{1}{\tilde{L}+\varsigma} e^{-2 \varsigma}+\int_{0}^{\tilde{L}} d \varsigma \varsigma^{2} \frac{1}{\tilde{L}-\varsigma} e^{-2 \varsigma}\right.\right. \\
\left.\left.\quad+\int_{\tilde{L}}^{\infty} d \varsigma \varsigma^{2} \frac{1}{\varsigma-\tilde{L}} e^{-2 \varsigma}\right]\right\} \rightarrow \alpha(Z \alpha) m_{e} c^{2}\left\{\frac{1}{\tilde{L}}-2\left[\int_{0}^{\infty} d \varsigma \varsigma^{2} \frac{1}{\tilde{L}+\varsigma} e^{-2 \varsigma}\right.\right.
\end{gathered}
$$




$$
\left.\left.+\int_{0}^{\tilde{L}-\varepsilon} d \varsigma \varsigma^{2} \frac{1}{\tilde{L}-\varsigma} e^{-2 \varsigma}+\int_{\tilde{L}+\varepsilon}^{\infty} d \varsigma \varsigma^{2} \frac{1}{\varsigma-\tilde{L}} e^{-2 \varsigma}\right]\right\}
$$

The principal difference from $S$-quantity consists in the presence of the logarithmically divergent one-dimensional Coulomb integrals in the first equality (the last two terms in the square brackets), because of which the small parameter $\varepsilon$, whose physical sense is explained below, was introduced into

$$
\begin{gathered}
K\left(Z_{N}>0\right)=\alpha(Z \alpha) m_{e} c^{2}\left\{\frac{1}{\tilde{L}}-2\left[\int_{0}^{\infty} d \varsigma \varsigma^{2} \frac{1}{\tilde{L}+\varsigma} e^{-2 \varsigma}+\left(-\tilde{L}^{2} e^{-2 \tilde{L}} \ln \mathcal{E}\right.\right.\right. \\
\left.\left.\left.+2 \int_{0}^{\tilde{L}} d \varsigma e^{-2 \varsigma} \varsigma(1-\varsigma) \ln (\tilde{L}-\varsigma)\right)+\left(-\tilde{L}^{2} e^{-2 \tilde{L}} \ln \varepsilon-2 \int_{\tilde{L}}^{\infty} d \varsigma e^{-2 \varsigma} \varsigma(1-\varsigma) \ln (\varsigma-\tilde{L})\right)\right]\right\}
\end{gathered}
$$

As can be seen, the form of the dependence $K(\tilde{L})$ for $\tilde{Z}_{N}(\equiv-\tilde{L})<0$ coincides with its form for $\tilde{Z}_{N}(\equiv \tilde{L})>0$ defined by these formulas, that is, $K\left(\tilde{Z}_{N}<0\right)$ and $K\left(Z_{N}>0\right)$ are in fact identical being functions of $\tilde{L}$ only without any additional restrictions on the electron states, as it should be according to their physical sense by analogy with $S$-quantity in these regions. Thus, the difference is also that the integration limits to obtain finite values of the corresponding integrals entering into the last expression.

After integration by parts, setting $\varepsilon \rightarrow 0$ where possible, we obtain

$$
\begin{gathered}
K(\tilde{L})=\alpha(Z \alpha) m_{e} c^{2}\left\{\frac{1}{\tilde{L}}-2 J_{1}+\frac{1}{2}+\tilde{L}+2 \tilde{L}^{2}\left(2 J_{2}-\ln \tilde{L}\right)-e^{-2 \tilde{L}}\left[1+3 \tilde{L}+4 \tilde{L}^{2}\left(J_{3}-\delta\right)\right]\right\} \\
J_{3} \equiv \int_{0}^{\infty} d \varsigma e^{-2 \varsigma} \ln \varsigma, \delta \equiv \ln \varepsilon<0
\end{gathered}
$$

The value of the integral $J_{3}<0$ can be calculated from formula (4.441.2) of [11]:

$$
J_{3}=-\frac{1}{2}(\ln 2+C)
$$

( $C \approx 0.577 \ldots$ is the Euler constant); the integrals in Eqs. (21) that cannot be calculated analytically are designated as

$$
J_{1} \equiv J_{1}(\tilde{L})=\int_{0}^{\infty} d \varsigma \frac{\varsigma^{2} e^{-2 \varsigma}}{\tilde{L}+\varsigma}, J_{2} \equiv J_{2}(\tilde{L})=e^{-2 \tilde{L}} \int_{0}^{\tilde{L}} d \varsigma e^{2 \varsigma} \ln \varsigma
$$

Analogous situation in the sense of the divergence of integrals is encountered in calculations of the energy of onedimensional two-electron atom or relativistic corrections to the energy of the two-dimensional hydrogen-like atom $[12,4]$. In these cases, the divergence was eliminated in principle with allowance for the deviation from the Coulomb law at small distances because of polarization of the electronpositron vacuum; moreover, in experimentally realized situation of one-dimensional orthohelium, it was reduced automatically [12].

In our case such reduction is lacking, and the deviation from the Coulomb potential with the necessity of introduction of the truncation parameter $\varepsilon$ can be caused by the following reasons.
Finite sizes of hydrogen-like nuclei with $\varepsilon \rightarrow \varepsilon_{N}=\frac{r_{N}}{a_{Z}}$, which in accordance with the generally accepted terminology is in fact equivalent in the simplest variant of taking into account the nucleus form-factor. Here $r_{N} \sim 10^{-13} \mathrm{~cm}$ is the order of magnitude of the nucleus radius, and in our designations $a_{Z}=\frac{\lambda_{C}}{Z \alpha}, \lambda_{C}=\frac{\hbar}{m_{e} c}$. Substituting numerical values, we obtain the estimation $\varepsilon_{N} \approx Z \times 10^{-5}$, and with logarithmic accuracy sufficient for our purposes, we can consider that the order of magnitude of the logarithmic truncation parameter $\delta=\ln \varepsilon$ in the second Eq. (22) is equal to $(-10)$.

To take into account polarization of vacuum, truncation 
should be performed for the dimensionless integration variable equal to $\varepsilon_{V A C}=(Z \alpha)$ [4]. Since $\left|\ln \varepsilon_{V A C}\right|<\left|\ln \varepsilon_{N}\right|$, the calculation error introduced by finite sizes of the nucleus will be greater than the electrodynamic error caused by polarization of the electron-positron vacuum, that is, in our calculations the latter can be disregarded, the more so that consideration of finite nucleus sizes does not influence our main results on the presence of a minimum in the region of negative values of the interaction energy and its asymptotic vanishing at infinity for a sufficiently wide range of variation of the $\varepsilon_{N}$, and in a general case - $\varepsilon$-value (see below) for the one-dimensional molecule $\mathrm{H}_{2}$.

There is also the third sufficiently exotic reason that necessitates the truncation to obtain finite results. As is well known, exactly in truly one-dimensional space the solution of the Poisson equation for the potential $\phi \rightarrow \phi_{1}$ has the form $\phi_{1} \sim|z|[13]$. By virtue of its non-physical character, if it is realized in experiments of the aforementioned type and if the given effect actually takes place in these experiments, it should be transformed in any way into the Coulomb dependence of the form $\sim 1 /|z|$ for the one-dimensional space being the subspace of the three-dimensional space at sufficiently small (and still unknown to us) distances from the nucleus, thereby introducing additional uncertainty in the truncation parameter.

In fact, this general uncertainty of the truncation parameter value for all above-indicated reasons does not matter for purposes of the present work whose conclusions referred to the one-dimensional molecule $\mathrm{H}_{2}$ are mainly qualitative in character, and this parameter can be considered as free with possible experimental determination of its value (see also Section 5). For vivid presentation, plots of the dimensionless interaction energy $\tilde{V}_{ \pm}^{( \pm)}$(see formulas (26-29) and comment below) as functions of $\tilde{L}$ are drawn in Figure 1 for $|\delta| \equiv|\ln \varepsilon|=5$ corresponding to the truncation parameter value $\varepsilon \sim 10^{-2}$ (this is caused by technical difficulties with the accepted presentation of the pairs of plots in each of Figures $1 \mathrm{a}$ and $1 \mathrm{~b}$; it seems likely that more realistic $|\delta|$ values lying in the interval (5-50) will not change our conclusions on the presence of a minimum and existence of the correct decreasing at $\infty$ asymptote (Eq. (31) for the functions $\tilde{V}_{+}^{(+)}$and $\tilde{V}_{-}^{(-)}$representing in our interpretation, as demonstrated below, physical variants of the interaction).

As can be seen from Eq. (21) (or Eqs. (26) and (28) below) and is confirmed by the results of concrete numerical calculations analogous to those shown in Figure 1, the depth of the negative minimum depends on the $\delta$-value, that is, on the value of the truncation parameter for reasonable values $\varepsilon<<1$ but not the fact itself of this minimum existence or vanishing of the asymptote at $\infty$ (in the latter case, the contribution of this truncation parameter to the asymptote, as well as of the entire expression in the square brackets in Eq. (28), is suppressed by the exponential factor $e^{-2 \tilde{L}}$ ). These conditions are obligatory for the existence of the onedimensional molecule $\mathrm{H}_{2}$ and in practice are not correlated with concrete value of the truncation parameter which, strictly speaking, is not known to us.

Calculation of the function $A$ presents no difficulties at all if we consider that in the one-dimensional variant the contribution of the first term of Eq. (13) is simply $\alpha(Z \alpha) m_{e} c^{2} \frac{S}{\tilde{L}}$, and the contribution of the second term is proportional to expression (16) for $S$ with the reverse sign and omission of the first factor in the integrand of Eq. (16).

Simple calculation with allowance for the values $P=P^{\prime}$ $= \pm 1$ previously established yields the result

$$
A(L) \rightarrow A^{( \pm)}(\tilde{L})=\alpha(Z \alpha) m_{e} c^{2}\left\{\frac{S^{( \pm)}}{\tilde{L}}-e^{-\tilde{L}}\left[1+\tilde{L} \pm \tilde{L}^{2}\right]\right\}
$$

with the dependence of this function on the parity, unlike the function $K$ given by Eq. (21) and analogous to Eq. (20).

Finally, using explicit expression (23) for the integral $J_{3}$, we obtain for the variants of the interaction energy $V_{S Y M}(R) \rightarrow$ $V_{ \pm}^{( \pm)}(L)$ of atoms in the one-dimensional molecule $H_{2}$ depending on the symmetry type (the low index " \pm ") of the coordinate part of the wave function of both electrons in the molecule and for identical values of parities $P=P^{\prime}= \pm 1$ (the upper index " \pm ") of the electron states in the atom the expressions in the form convenient for numerical calculations

$$
\begin{gathered}
V_{ \pm}^{( \pm)} \equiv \alpha(Z \alpha) m_{e} c^{2} \tilde{V}_{ \pm}^{( \pm)}, \tilde{V}_{ \pm}^{( \pm)}=\frac{\tilde{K} \pm \tilde{A}^{( \pm)}}{1 \pm S^{( \pm)}} \\
S^{( \pm)}=e^{-\tilde{L}}\left(1+\tilde{L} \pm \frac{1}{3} \tilde{L}^{3}\right) \\
\tilde{K}=\frac{1}{\tilde{L}}-2 J_{1}+\frac{1}{2}+\tilde{L}+2 \tilde{L}^{2}\left(2 J_{2}-\ln \tilde{L}\right)+e^{-2 \tilde{L}}\left[-1-3 \tilde{L}+2 \tilde{L}^{2}(\ln 2+C-2|\delta|)\right]
\end{gathered}
$$




$$
\begin{gathered}
J_{1}=\int_{0}^{\infty} d \varsigma \frac{\varsigma^{2} e^{-2 \varsigma}}{\tilde{L}+\varsigma}, J_{2}=e^{-2 \tilde{L}} \int_{0}^{\tilde{L}} d \varsigma e^{2 \varsigma} \ln \varsigma \\
\tilde{A}^{( \pm)}=e^{-\tilde{L}}\left(\frac{1}{\tilde{L}}-\tilde{L} \mp \frac{2}{3} \tilde{L}^{2}\right)
\end{gathered}
$$

It is easy to be convinced that with accuracy necessary for an analysis of the asymptote for $\tilde{L} \rightarrow 0$, we have the following expansions:

$$
\left.\tilde{A}^{( \pm)}\right|_{\tilde{L} \rightarrow 0} \rightarrow \frac{1}{\tilde{L}}-1-\frac{1}{2} \tilde{L}+\ldots,\left.\tilde{K}\right|_{\tilde{L} \rightarrow 0} \rightarrow \frac{1}{\tilde{L}}-1+\ldots,\left.S^{( \pm)}\right|_{\tilde{L} \rightarrow 0} \rightarrow 1-\frac{1}{2} \tilde{L}^{2}+\ldots
$$

(in $\tilde{K}$ and $S^{( \pm)}$the contribution linear in $\tilde{L}$ is reduced, and the parity of the state does not contribute to this asymptote).

Then in the asymptote $\tilde{L} \rightarrow 0$ for the one-dimensional hydrogen molecule, taking into account Eqs. (26-29) for all interaction variants we obtain

$$
V_{+}^{( \pm)}, V_{-}^{( \pm)} \rightarrow \frac{e^{2}}{a_{1} \tilde{L}}=\frac{e^{2}}{L}
$$

as it should be since at small distances only the nuclei interact [6]. In this case, this limit for $V_{+}^{( \pm)}$is trivial, since the contribution yields only the main order, whereas for

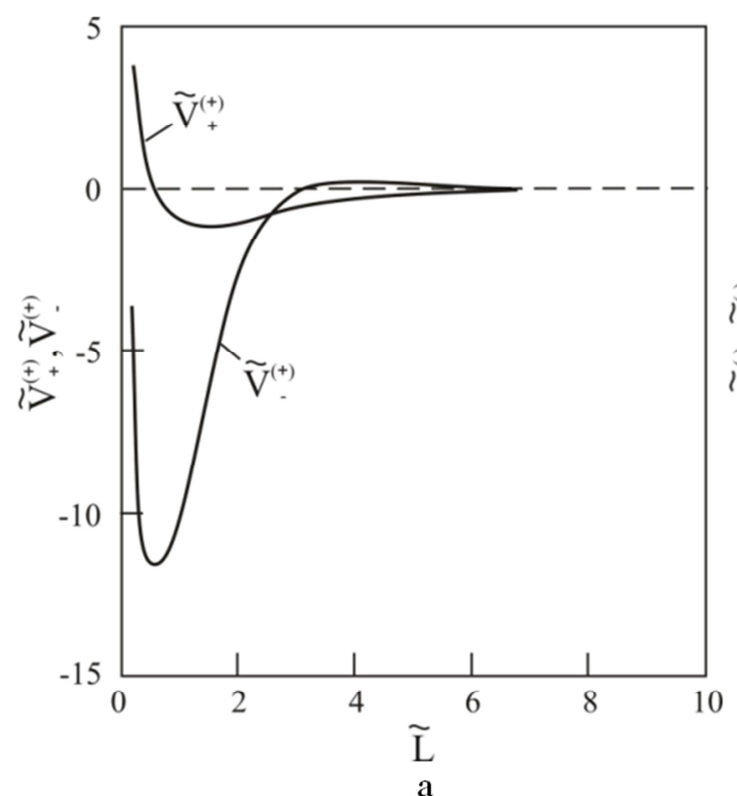

$V_{-}^{( \pm)}$terms of higher order should be taken into account in expansions of functions $\tilde{A}^{( \pm)}, \tilde{K}$, and $S^{( \pm)}$).

From the plots of functions $\tilde{V}_{ \pm}^{( \pm)}$shown in Figure 1 it can also be seen that qualitatively correct dependence on the distance between the nuclei in the molecule, coinciding with the three-dimensional one, including the asymptote of the form

$$
\left.\tilde{V}_{ \pm}^{( \pm)}\right|_{\tilde{L} \rightarrow \infty} \rightarrow-0
$$

and the presence of the minimum in the region of negative values, is realized in the interaction variants $\tilde{V}_{+}^{(+)}$and $\tilde{V}_{-}^{(-)}$.

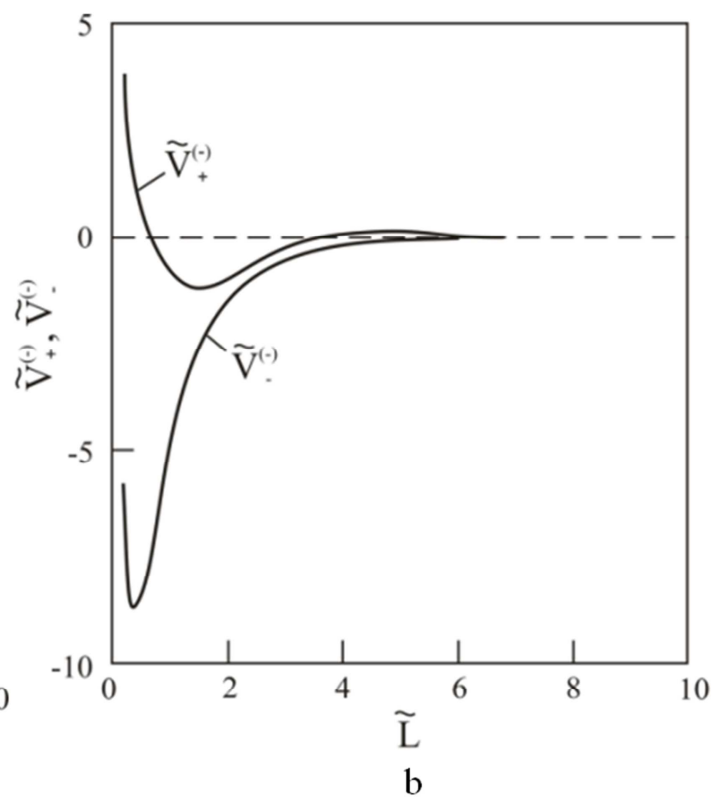

Figure 1. Plots of functions $\tilde{V}_{+}^{(+)}, \tilde{V}_{-}^{(+)}(a)$ and $\tilde{V}_{-}^{(-)}, V_{+}^{(-)}$(b), appearing in general formula (26), depending on the dimensionless distance $\tilde{L}$.

Meanwhile, for $\tilde{V}_{-}^{(+)}$and $\tilde{V}_{+}^{(-)}$, in the right branches of the plots there exist regions in which these functions for $|\delta|$ $\sim(>) 5$ are greater than zero, though insignificantly $(\sim(<)$ $10^{-2}$ ) -Figure 1, with the subsequent asymptote of the form $\rightarrow+0$ for $\tilde{L} \rightarrow \infty$, that is, it seem likely that the nonphysical effect of repulsion arises; this means that for this reason, these variants in our approach to the problem of the one-dimensional molecule cannot be realized, despite the presence of the negative minimum $\tilde{V}_{-}^{(+)}, \tilde{V}_{+}^{(-)}<0$ and the correct asymptote given by Eq. (30) at the zero point.

It should also be noted that in the two-dimensional (Section 4) or three-dimensional cases, the physical variant of the interaction with the low index "-" is absent at all, unlike the existence of the one-dimensional variant $\tilde{V}_{-}^{(-)}$ 
(and probably $\tilde{V}_{-}^{(+)}$) established above $[6,7]$.

Thus, it seems most likely that in the one-dimensional hydrogen molecule in the state with the symmetric electron wave function, the parities of the electron state should be positive (this is the $\tilde{V}_{+}{ }^{+}$interaction variant), and with the asymmetric electron wave function, they should be negative (variant $\tilde{V}_{-}^{(-)}$). The physical reason for this remained unclear to us together with the question whether the interaction variants $\tilde{V}_{-}^{(+)}$and $\tilde{V}_{+}^{(-)}$can be realized in principle.

As can be seen, asymptote (31) decreasing at $\infty$ for physical interaction variants $\tilde{V}_{+}^{(+)}$and $\tilde{V}_{-}^{(-)}$, which cause no doubt, is possible only if the following limit does exist of the specific combination of terms in Eq. (28):

$$
\lim _{\tilde{L} \rightarrow \infty}\left\{\frac{1}{\tilde{L}}-2 J_{1}+\frac{1}{2}+\tilde{L}+2 \tilde{L}^{2}\left(2 J_{2}-\ln \tilde{L}\right)\right\} \rightarrow-0
$$

This circumstance, taking into account the form of the characteristic integral $J_{2}$ given by Eq. (24) appearing, as well as the integral $J_{3}$ given by Eq. (23), in the course of transformation of the diverging integrals by introduction of the truncation parameter (the integral $J_{1}$ enters also into the intermediate expressions before the truncation procedure), can hardly be random, and with a high probability confirms the adequacy of the method of eliminating the divergence of the one-dimensional integrals used by us and conclusions of the present work concerning the one-dimensional molecule $\mathrm{H}_{2}$. The same is true for the existence of asymptote (30) for all interaction variants that follows from the expansions of functions $S^{( \pm)}(\tilde{L}), \tilde{K}(\tilde{L}), \tilde{A}^{( \pm)}(\tilde{L})$ for $\tilde{L} \rightarrow 0$ presented above.

From this Section it follows with confidence that the one-

$$
\begin{gathered}
\tilde{\psi}=R_{N|m|}(\rho) \Phi_{m}(\phi), \rho=\frac{r}{a_{Z}} \\
R_{N|m|}(\rho)=C_{N|m|} \frac{(2 \lambda \rho)^{|m|}}{(2|m|) !} e^{-\lambda \rho} F(-N+|m|, 2|m|+1 ; 2 \lambda \rho) \\
\lambda=\frac{1}{(N+1 / 2)}, C_{N|m|}=\sqrt{2 \lambda^{3} \frac{(N+|m|) !}{(N-|m|) !}} ; N \geq|m|=0,1, \ldots \\
\Phi_{m}=\frac{1}{\sqrt{2 \pi}} e^{i m \phi}, m=0, \pm 1, \ldots
\end{gathered}
$$

with the energy

$$
E \equiv E_{N}=-\frac{(Z \alpha)^{2} m_{e} c^{2}}{2(N+1 / 2)^{2}}
$$

Here $F$ is the degenerate hypergeometric function.

In the ground state $N=0$ we have

$$
\tilde{\psi} \rightarrow \tilde{\psi}_{0}(\rho)=\sqrt{\frac{8}{\pi}} e^{-2 \rho}
$$

Apparently, the function $S$ for the flat Molecule $\mathrm{H}_{2}$ is

$$
S \equiv S(\tilde{R})=\frac{8}{\pi} \int_{0}^{\infty} d \rho \rho \int_{0}^{2 \pi} d \phi e^{-2 \rho-2 \sigma}
$$




$$
\sigma=\sqrt{\rho^{2}+\tilde{R}^{2}-2 \rho \tilde{R} \cos \phi}, \tilde{R}=\frac{R}{a_{Z}}
$$

The function $K$ can be written in the form

$$
K=\alpha(Z \alpha) m_{e} c^{2}\left[\frac{1}{\tilde{R}}-\frac{8}{\pi} \int_{0}^{\infty} d \rho \rho \int_{0}^{2 \pi} d \phi \frac{1}{\sigma} e^{-4 \rho}\right]
$$

The function $A$ can also be represented in the similar form:

$$
A=\alpha(Z \alpha) m_{e} c^{2}\left[\frac{S}{\tilde{R}}-\frac{8}{\pi} \int_{0}^{\infty} d \rho \int_{0}^{2 \pi} d \phi e^{-2 \rho-2 \sigma}\right]
$$

The final result for the interaction energy of atoms in the flat molecule $H_{2}$ can be written by analogy with Eq. (26) in the form also convenient for numerical calculations:

$$
V_{ \pm}=\alpha(Z \alpha) m_{e} c^{2} \tilde{V}_{ \pm}(\tilde{R}), \tilde{V}_{ \pm}(\tilde{R})=\frac{\tilde{K} \pm \tilde{A}}{1 \pm S}
$$

where now

$$
\begin{gathered}
S=\frac{8}{\pi} \int_{0}^{\infty} d \rho \rho \int_{0}^{2 \pi} d \phi e^{-2 \rho-2 \sigma} \\
\tilde{K}=\frac{1}{\tilde{R}}-\frac{8}{\pi} \int_{0}^{\infty} d \rho \rho \int_{0}^{2 \pi} d \phi \frac{1}{\sigma} e^{-4 \rho} \\
\tilde{A}=\frac{S}{\tilde{R}}-\frac{8}{\pi} \int_{0}^{\infty} d \rho \int_{0}^{2 \pi} d \phi e^{-2 \rho-2 \sigma}
\end{gathered}
$$

For $\tilde{R} \rightarrow 0$, by analogy with Eq. (30), we have

$$
V_{+} \rightarrow \frac{e^{2}}{a_{1} \tilde{R}}=\frac{e^{2}}{R}
$$

In this variant for $V_{+} \sim \tilde{V}_{+}$, as it follows from the result of numerical calculation and from the plot shown in Figure 2, another physical asymptote $\left.\tilde{V}\right|_{\tilde{R} \rightarrow \infty} \rightarrow-0$ mentioned in Section 3 is observed simultaneously with the presence of the minimum in the region $\tilde{V}_{+}<0$, by analogy with the threedimensional case [6].

This means that the two-dimensional hydrogen molecule can exist as, naturally, the three-dimensional molecule. Moreover, as demonstrated in Section 5, the energy characteristics, like the binding energy, can be calculated, unlike the one-dimensional case (see Section 5 also). The variant $V_{-} \sim \tilde{V}_{-}>0$, by analogy with the three-dimensional case, cannot be realized [6].

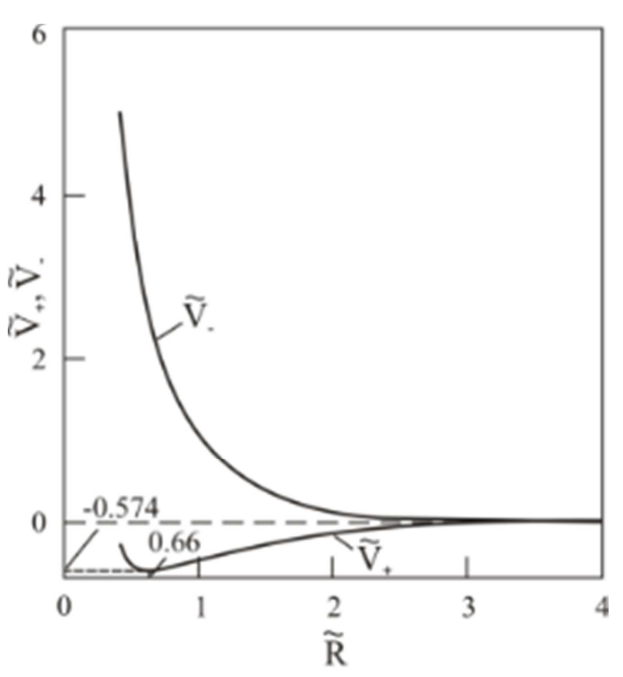

Figure 2. Plots of functions $\tilde{V}_{ \pm}$given by Eq. (43) depending on the dimensionless distance between the nuclei $\tilde{R}$.

\section{Energies of the Two-Dimensional and One-Dimensional Hydrogen Molecules}

Obviously, the total energy of the two-dimensional molecule in the ground state with physical interaction variant $V_{+}$as a function of the variation parameter $Z^{\prime}$ is equal to

$$
E_{m o l}\left(Z^{\prime}\right)=2\langle\hat{T}\rangle_{Z^{\prime}}{ }^{(0)}+2\left\langle\hat{\Pi}_{Z}\right\rangle_{Z^{\prime}}{ }^{(0)}+V_{+}
$$

Here $\langle\hat{T}\rangle_{Z^{\prime}}{ }^{(0)}$ and $\left\langle\hat{\Pi}_{Z}\right\rangle_{Z^{\prime}}{ }^{(0)}$ are the average kinetic and potential energies of the ground state $N=0$ of the electron in the two-dimensional hydrogen-like atom calculated with usage of function (38) and with allowance for Eq. (8-10), which according also to general relations (A17, A19), equal to 


$$
\langle\hat{T}\rangle_{Z^{\prime}}{ }^{(0)}=2\left(Z^{\prime} \alpha\right)^{2} m_{e} c^{2},\left\langle\hat{\Pi}_{Z}\right\rangle_{Z^{\prime}}{ }^{(0)}=-4(Z \alpha)\left(Z^{\prime} \alpha\right) m_{e} c^{2}
$$

Here $V_{+}$is given by the first formula (43) after the substitution $Z \rightarrow Z^{\prime}: V_{+} \rightarrow \alpha\left(Z^{\prime} \alpha\right) m_{e} c^{2} \times \tilde{V}_{+}(\tilde{R})$

Thus, we obtain

$$
E_{m o l}\left(Z^{\prime}\right)=4 \alpha^{2} m_{e} c^{2}\left[Z^{\prime 2}-2 Z Z^{\prime}+Z^{\prime} \tilde{V}_{+}(\tilde{R})\right]
$$

The standard variation procedure (see also the end of this Section) $[6,7]$

$$
\frac{\partial E_{m o l}}{\partial Z^{\prime}}=0 \rightarrow Z^{\prime} \equiv Z_{e f f}=Z-\frac{1}{2} \tilde{V}_{+}(\tilde{R})
$$

yields the following result for the energy of the two-dimensional molecule

$$
E_{m o l} \equiv E_{m o l}\left(Z_{e f f}\right)=-4 \alpha^{2} m_{e} c^{2}\left[Z-\frac{1}{2} \tilde{V}_{+}(\tilde{R})\right]^{2}
$$

Analogous result (in accordance with the material presented in the Appendix) for the one-dimensional molecule with strictly fixed interaction variants $\tilde{V}_{+}^{(+)}$and $\tilde{V}_{-}^{(-)}$has the form

$$
E_{m o l} \equiv E_{m o l}\left(Z_{e f f}\right)=-\alpha^{2} m_{e} c^{2}\left[Z-\frac{1}{2}\left\{\tilde{V}_{+}^{(+)}(\tilde{L}), \tilde{V}_{-}^{(-)}(\tilde{L})\right\}\right]^{2}
$$

Moreover, in the ground state of molecules in Eq. $(52,53)$, the values $\tilde{V}<0$ should be taken at points of minima of the plots shown in Figures 1 and 2 for these physical interaction variants (this could be done at once in initial expressions (4951); this changes nothing). Namely,

In the ground state of the two-dimensional molecule in Eq. (52) we should take the value $\tilde{V}_{+}<0$ at the point of the minimum of the lower plot in Figure 2. Numerical calculation yields the following value of the function $\tilde{V}_{+}(\tilde{R})$ (indicated in Figure 2 also) in the minimum for the dimensionless distance $\tilde{R} \rightarrow \tilde{R}_{e q} \approx 0.660$ corresponding to the equilibrium ground state of the two-dimensional molecule $H_{2}: \tilde{V}_{+}(\tilde{R}) \rightarrow \tilde{V}_{e q} \approx-0.574$. This is by about 10 times higher than the corresponding value for the threedimensional molecule $(\approx-0.0646)$ [6]. Thus, the distance between the nuclei in the two-dimensional molecule is $R_{e q} \approx$ $0.35 \times 10^{-8} \mathrm{~cm}$. Note that in the three-dimensional molecule, it is by about three times greater [6].

From Eq. (52), the value of the ground state energy $E_{\text {mol.eq. }}$ for the above-indicated value of $\tilde{V}_{e q}$ and $Z=1$ is

$$
E_{\text {mol.eq. }} \approx-6,6 \alpha^{2} m_{e} c^{2} \approx-180 \mathrm{eV}
$$

The corresponding result obtained under assumption that the perturbation theory is valid when

$$
E_{\text {mol.eq. }}=2 E_{0}+V_{+}\left(R_{e q}\right), V_{+}\left(R_{e q}\right)=\alpha^{2} m_{e} c^{2} \tilde{V}_{+}\left(\tilde{R}_{e q}\right)
$$

with the $E_{0}$ value given by Eq. (37) at $N=0$ and the above-mentioned value $\tilde{V}_{+}\left(\tilde{R}_{e q}\right) \equiv \tilde{V}_{e q} \approx-0.574$ is

$$
E_{\text {mol.eq. }} \approx-4.574 \alpha^{2} m_{e} c^{2} \approx-124 \mathrm{eV}
$$

Possibly, it is necessary to consider the result given by Eq. (54) as more adequate in view of the formal inapplicability of the perturbation theory (the energy $2 E_{0}$ of the molecule disregarding the atomic interaction energy $V_{+}$has the same order in $\alpha$ as the last). However, in other cases, the situation can be observed when both approaches yield almost identical results as, for example, in calculation of the energy of the three-dimensional helium atom, but in our case it not so[6,7]

Obviously, the binding energy of atoms $E_{\text {bind.en. }}$ in the two-dimensional molecule $\mathrm{H}_{2}$ within the limits of the variation method is $E_{\text {bind.en. }}=2 E_{0}-E_{\text {mol.eq. }}$ with $E_{\text {mol.eq. }}$ value given by Eq. (54). This yields

$$
E_{\text {bind.en. }} \approx 2.6 \alpha^{2} m_{e} c^{2} \approx 71 \mathrm{eV}
$$

which is by about 31 times higher than the corresponding theoretical result $(\approx 2.25 \mathrm{eV})$ and by 25 times higher than the experimental value $(\approx 2.8 \mathrm{eV})$ for the threedimensional molecule [6].

According to the perturbation theory, the binding energy is

$$
E_{\text {bind.en. }}=\left|V_{+}\right| \rightarrow \alpha^{2} m_{e} c^{2}\left|\tilde{V}_{e q}\right| \approx 0.574 \alpha^{2} m_{e} c^{2} \approx 16 \mathrm{eV}
$$


In such a manner, it can be stated that the binding energy for the two-dimensional molecule in any case is always much higher (at least, approximately by an order of magnitude) than for the three-dimensional molecule.

As to the one-dimensional molecule $\mathrm{H}_{2}$, in this stage we cannot make theoretical predictions on the numerical value of the energy of such molecule and its binding energy because these values, by analogy with values of the functions $\tilde{V}_{+}^{(+)}$ $(\tilde{L})$ and $\tilde{V}_{-}^{(-)}(\tilde{L})$ of the presupposed physical interaction variants at the point of minimum with the equilibrium distance $\tilde{L} \rightarrow \tilde{L}_{e q}$ depend, as already demonstrated in Section 3 , on the value of the truncation parameter used in analytical and numerical calculations. This parameter, as already mentioned above, is in fact simultaneously free, because for the known experimental data on the onedimensional molecules $\mathrm{H}_{2}$, it is possible to adjust such value that the results of theoretical calculations would be closest to them (this is also in agreement with the approximate character and the idea of the variation method itself).

Namely, the binding energy of the one-dimensional molecule $\mathrm{H}_{2}$ within the framework of the variation method for the known equilibrium dimensionless distance $\tilde{L}_{e q}$ between the nuclei for the above-indicated physical interaction variants is equal to $E_{\text {bind.en. }}=2 E_{1}-E_{\text {mol.eq. }}$, where $E_{1}$ is given by formula (15), and the value of $E_{\text {mol.eq. }}$. is obtained from formula (53) at $Z=1$ with the functions $\tilde{V}_{+}^{(+)}$and $\tilde{V}_{-}^{(-)}$given by Eqs. (26-29) for $\tilde{L} \rightarrow \tilde{L}_{e q}$. Obviously, according to the perturbation theory, the $E_{\text {bind.en. }}{ }^{-}$ value assumes the form $E_{\text {bind.en. }}=\left|V_{+}^{(+)}\right|,\left|V_{-}^{(-)}\right|$when $\tilde{L}$ $\rightarrow \tilde{L}_{e q}$.

If the $E_{\text {bind.en. }}$ - value, by analogy with the $\tilde{L}_{e q}$ - value, is determined experimentally, by adjusting the variation parameter $\varepsilon<<1$ that influences, as already mentioned above, on the depth of the potential well, but has no effect on the correct decreasing asymptote at $\infty$ given by Eq. (31) the quantitative agreement can be obtained with the experiment within the framework of our theory of the one-dimensional molecule $\mathrm{H}_{2}$ at least in one of the two above-mentioned methods of calculating $E_{\text {bind.en. }}$. At the same time, this would also provide useful information on relative roles of reasons for the deviation from the Coulomb interaction at small distances considered in Section 3.

Thus, our theory of one-dimensional molecules $\mathrm{H}_{2}$ is much less complete than the two-dimensional one (and simultaneously more cumbersome because of the procedure of regularization of the diverging integrals). However, we can reliably state that they, as well as two-dimensional molecules, exist, since from our numerical calculations it follows that all necessary conditions are satisfied, including physical asymptotes at the zero point and at infinity and the presence of the minimum for negative values of the interaction energy of atoms in molecules for the established physical interaction variants; moreover, for both onedimensional and two-dimensional molecules, $\left.\tilde{V}\right|_{\tilde{L}, \tilde{R} \rightarrow \infty} \rightarrow$ -0 , as most likely expected by analogy with the threedimensional molecule [6, 7].

Further, we must note also that when using variation method we have not considered $Z$-value, which enters into definitions of the dimensionless distances $\tilde{R}=R / a_{Z}$ and $\tilde{L}=L / a_{Z}$ depending on this $Z$ through the parameter $a_{Z}$ given by Eq. (10), as a variation parameter. The reason is the following.

As it follows from Eq. (50), in the variation equation $\frac{d E_{m o l}}{d Z^{\prime}}=0 \quad$ so obtained the corresponding additional contribution has the form $\sim Z^{\prime} \frac{d \tilde{V}_{+}}{d \tilde{R}} \times\left.\frac{d \tilde{R}}{d Z}\right|_{Z \rightarrow Z^{\prime}}$. However, as mentioned above, to obtain numerical results given by Eqs. (54) and (56), we must take the value of $d \tilde{V}_{+} / d \tilde{R}$, as well as expression (50), at the point of the minimum at $\tilde{R}=\tilde{R}_{e q}$, at which it vanishes. Thus, in the case under consideration it is quite correct to take into account only the explicit dependence on $Z^{\prime}$ in Eq. (50) using first Eq. (51): $\frac{\partial E_{m o l}}{\partial Z^{\prime}}=$ 0 .

Obviously, the same reasons for the existence of the molecule and the applicability of the variation method, but without indication of the value of the truncation parameter $\varepsilon$ and unknown concrete numerical values of $\tilde{L}_{e q}, \tilde{V}_{e q}$ in the established physical interaction variants are also applicable to the one-dimensional molecule.

\section{Discussion}

As follows from the result presented in Section 5, the binding energy of atoms in the two-dimensional molecule should be considerably (up to an order of magnitude) higher than in the three-dimensional molecule. Since in experiments the transition of the three-dimensional sodium Bose condensate to the two-dimensional (as well as to onedimensional) Bose condensate was observed, in the same experiment with molecules $\mathrm{H}_{2}$, the two-dimensional Bose condensate of molecules $\mathrm{H}_{2}$ is fairly probable, since the state with a larger fraction of the two-dimensional phase will have lower energy of the condensate [1]. In this case, it will be possible to test the results presented here, for example, for the binding energy, similar to the conventional threedimensional molecules [6].

According to the conclusions of the present work, the onedimensional Bose condensate of molecules $\mathrm{H}_{2}$ must also manifest itself, and determination of its binding energy would be rather desirable, as noted above, to complete our 
theory of these molecules. It seems likely also that it is possible to obtain the one-dimensional molecules in the experiment analogous to that reported in [2].

More certain conclusions about this subject would be difficult because of the limited volume of the available experimental information.

\section{Appendix}

On Average Values of the Kinetic and Potential Energy Operators in the Hydrogen-Like Atom

Let us preliminary find the average values of the potential and kinetic energy operators $\hat{\Pi}_{Z}=-\frac{\left(Z e^{2}\right)}{r}, \hat{\mathrm{T}}=-\frac{\hbar^{2}}{2 m_{e}} \Delta$ of electron for states with any arbitrary set of quantum numbers $\{n, l, m\}$ and in the most real variant of threedimensional ( $D=3$ ) hydrogen-like atom. In this connection, we emphasize that because of lacking of these calculations in the literature known to us, the material presented in the Appendix, in our opinion, is also of independent methodical interest and can be added to the conventional course of quantum mechanics. In this case, the corresponding results for the one-dimensional $(D=1)$ and two-dimensional ( $D=$ 2 ) atoms, being one of the accompanying objects of consideration in the present work, are derived automatically.

We will be sticking to the interpretation of the authors of $[6,7]$. According to this authors the necessary element of the variation method as applied to the dimensionless nucleus charge $Z$ (designated by $Z^{\prime}$ in the wave functions $\psi \rightarrow$ $\left.\psi^{\prime}\right)$ is calculation of the average values of the potential and kinetic electron energy operators $\hat{\Pi}_{Z}, \hat{\mathrm{T}}$ in the field of the nucleus $\left(Z^{\prime} e\right)$ in terms of these functions determined from the Schrödinger equation

$$
\left(\hat{\mathrm{T}}+\hat{\Pi}_{Z^{\prime}}\right) \psi^{\prime}=E^{\prime} \psi^{\prime}
$$

We designate these average values by $\left\langle\hat{\Pi}_{Z}\right\rangle_{Z^{\prime}}$ and $\langle\hat{\mathrm{T}}\rangle_{Z^{\prime}}$, respectively, and $E^{\prime} \sim Z^{\prime 2}$-quantity in Eq. (A1) was obtained from $E \sim Z^{2}$ appearing below in Eq. (A4) after substitution $Z \rightarrow Z^{\prime}$.

In this case, for the ground state $n=1$ of the atom in the three-dimensional ( $D=3$ ) space, as has long been known, the equalities hold true [6]

$$
\begin{aligned}
& \left.\left.\left\langle\hat{\Pi}_{Z}\right\rangle_{Z} \equiv\left\langle\hat{\Pi}_{Z}\right\rangle_{Z^{\prime}}\right|_{Z^{\prime} \rightarrow Z}=2 E \quad \text { (A2) } \quad Z \rightarrow Z^{\prime} \text { in Eq. (10) for } a_{Z}\right) \text { is } \\
& \left\langle\hat{\Pi}_{Z}\right\rangle_{Z^{\prime}}=-(Z \alpha)\left(Z^{\prime} \alpha\right) m_{e} c^{2} C_{n l} \int_{0}^{\infty} d \rho \rho e^{-(2 \rho / n)}\left(\frac{2 \rho}{n}\right)^{2 l} \times F^{2}\left(-n+l+1 ; 2 l+2 ; \frac{2 \rho}{n}\right)
\end{aligned}
$$

$$
\left.\langle\hat{\mathrm{T}}\rangle_{Z} \equiv\langle\hat{\mathrm{T}}\rangle_{Z^{\prime}}\right|_{Z^{\prime} \rightarrow Z}=|E|
$$

As already mentioned above, no proof of Eqs. (A2, A3) for any arbitrary sets of quantum numbers $\{n, l, m\}$ can be found in the literature.

In formulas (A2, A3), $E$ is the electron energy of the hydrogen-like (one-electron) atom depending on the energy quantum number $n$. As is well known, for $D=3$ it is

$$
E \equiv E_{n}=-\frac{(Z \alpha)^{2} m_{e} c^{2}}{2 n^{2}}, n=1,2, \ldots
$$

Let us further find the above-mentioned average values $\left\langle\hat{\Pi}_{Z}\right\rangle_{Z^{\prime}}$ and $\langle\hat{\mathrm{T}}\rangle_{Z^{\prime}}$ by direct calculation with corresponding generalization of formulas (A2, A3) to any states with the set of quantum numbers $\{n, l, m\}$ in $D=3$. In this case, it is not required to consider separately subspaces $D=1,2$ of the three-dimensional space because the scheme of reasoning for them is absolutely analogous to the case $D=3$ (see also about it below).

Let us find at first $\left\langle\hat{\Pi}_{Z}\right\rangle_{Z^{\prime}}$. The normalized threedimensional radial function required for calculation in terms of the degenerate hypergeometric function $F$ can be found, for example, in [7]:

$$
\begin{gathered}
R_{n l}(r)=a_{Z}^{-3 / 2} R_{n l}(\rho), \rho=\frac{r}{a_{Z}} \\
R_{n l}(\rho)=C_{n l} e^{-\frac{\rho}{n}\left(\frac{2 \rho}{n}\right)^{l} F\left(-n+l+1 ; 2 l+2 ; \frac{2 \rho}{n}\right)} \\
C_{n l}=\frac{2}{n^{2}(2 l+1) !} \sqrt{\frac{(n+l) !}{(n-l-1) !}}
\end{gathered}
$$

With the potential energy operator expressed through the dimensionless variable $\rho$

$$
\hat{\Pi}_{Z}=-\frac{\left(Z e^{2}\right)}{r} \equiv-\frac{1}{a_{Z}} \frac{\left(Z e^{2}\right)}{\rho}
$$

$\left\langle\hat{\Pi}_{Z}\right\rangle_{Z^{\prime}}$-value in terms of the function given by Eq. (A5) with allowance for Eq. (A6) (and with the substitution
To calculate the integral over $d \rho$, we take advantage of formula (7.622.1) from reference book [11]. In this book the integral appearing in it is expressed through the hypergeometric series of general form $F(b, a ; d ; z)$ : 


$$
I(s, a, b, d, \lambda) \equiv \int_{0}^{\infty} d t e^{-s t} t^{d-1} F(b ; d ; t) F(a ; d ; \lambda t)=\Gamma(d) \times(s-1)^{-b}(s-\lambda)^{-a} s^{a+b-d} \quad F\left(b, a ; d ; \frac{\lambda}{(s-1)(s-\lambda)}\right)
$$

To avoid the intersection with already introduced standard designations, we have replaced the original parameters $\alpha, a, c$ by the following: $\alpha, a, c \rightarrow a, b, d$, respectively [11].

In our case, $a=b=-n+l+1, d=2 l+2, \lambda=1, s=1$, and because of singular structure of expression (A10), including the case of $\lambda=1$, it is impossible to set at once $s=1$, but it is necessary to calculate the corresponding limiting value; after replacement of the integration variable $t=\frac{2 \rho}{n}$ in integral (A9), we obtain with allowance for Eqs. (A9) and (A10):

$$
\left\langle\hat{\Pi}_{Z}\right\rangle_{Z^{\prime}}=-(Z \alpha)\left(Z^{\prime} \alpha\right) m_{e} c^{2} C_{n l}^{2}\left(\frac{n}{2}\right)^{2} \lim _{s \rightarrow 1} I(s,-n+l+1,-n+l+1,2 l+2,1)
$$

Taking into account the form of the generalized hypergeometric series

$$
F(b, a ; d ; z)=1+\frac{a b}{d} \frac{z}{1 !}+\frac{b(b+1) a(a+1)}{d(d+1)} \frac{z^{2}}{2 !}+\cdots+\frac{[b(b+1) \ldots(b+k)][a(a+1) \ldots(a+k)]}{d(d+1) \ldots(d+k)} \times \frac{z^{k+1}}{(k+1) !}+\ldots
$$

we obtain that in the limit $s \rightarrow 1$ the nonzero contribution to Eq. (A11) gives only the last term of series (A12), finite in our case, with the value $k=n-l-2$, so that

$$
\lim _{s \rightarrow 1} I(s,-n+l+1,-n+l+1,2 l+2,1)=\frac{[(n-l-1) !]^{2}}{(2 l+2) \ldots(n+l)} \times \frac{1}{(n-l-1) !}=\frac{(n-l-1) !(2 l+1) !}{(n+l) !}
$$

Taking into account formula (A7) for the normalization coefficient $C_{n l}$, we obtain from Eqs. (A11) and (A13):

$$
\left\langle\hat{\Pi}_{Z}\right\rangle_{Z^{\prime}}=-(Z \alpha)\left(Z^{\prime} \alpha\right) m_{e} c^{2} \frac{1}{n^{2}}
$$

Thus, with allowance for the first equality (A2) and expression (A4) for the energy, we obtain

$$
\left\langle\hat{\Pi}_{Z}\right\rangle_{Z}=-\frac{(Z \alpha)^{2} m_{e} c^{2}}{n^{2}}=2 E_{n}
$$

On the other hand, from the Schrödinger equation (A1) after replacement $Z^{\prime} \rightarrow Z$ in it, we have the quantum variant of the total energy expression $E_{n}=\langle\hat{\mathrm{T}}\rangle_{Z}+\left\langle\hat{\Pi}_{Z}\right\rangle_{Z}$, and with allowance for Eq. (A15) $\langle\hat{\mathrm{T}}\rangle_{Z}=-E_{n}=\left|E_{n}\right|$; as a consequence, we also obtain

$$
\left.\langle\hat{\mathrm{T}}\rangle_{Z^{\prime}} \equiv\langle\hat{\mathrm{T}}\rangle_{Z}\right|_{Z \rightarrow Z^{\prime}}=\frac{\left(Z^{\prime} \alpha\right)^{2} m_{e} c^{2}}{2 n^{2}}
$$

Thus, it is not necessary to calculate the average value $\langle\hat{\mathrm{T}}\rangle_{Z^{\prime}}$, with the explicit form of the kinetic energy operator $\hat{\mathrm{T}}=-\frac{\hbar^{2}}{2 m_{e}} \Delta$, because accompanying relations (A2,

have already been proved for the examined case.

The same relations (A14-A16) and (A2, A3) for the onedimensional ( $D=1$ ) atom can be derived absolutely analogously using the known solution of the Schrödinger equation $[7,10]$. For the two-dimensional $(D=2)$ atom they can be derived using Eqs. (33-38) after replacement of the expression for energy (Eq. (A4)) by Eq. (37) (see also Eqs. (A17-A19) below).

This is not surprising, since the one-dimensional and twodimensional wave functions are derived, as is well known from the three-dimensional functions given by Eqs. (A5-A7) by means of the corresponding transformation of the threedimensional radial Schrödinger equation ; moreover, in the two-dimensional case with replacement of the set of quantum numbers $\{n, l, m\} \rightarrow\{N, m\}$, and in the one-dimensional case with replacement $\{n, l, m\} \rightarrow\{n, P\}[10,15,14,7]$.

In particular, in this two-dimensional case, we have

$$
\begin{gathered}
\left\langle\hat{\Pi}_{Z}\right\rangle_{Z^{\prime}}=-(Z \alpha)\left(Z^{\prime} \alpha\right) m_{e} c^{2} \frac{1}{(N+1 / 2)^{2}} \\
\left\langle\hat{\Pi}_{Z}\right\rangle_{Z}=-\frac{(Z \alpha)^{2} m_{e} c^{2}}{(N+1 / 2)^{2}}=2 E_{N} \\
\langle\hat{\mathrm{T}}\rangle_{Z^{\prime}}=\frac{\left(Z^{\prime} \alpha\right)^{2} m_{e} c^{2}}{2(N+1 / 2)^{2}},\langle\hat{\mathrm{T}}\rangle_{Z}=\left|E_{N}\right|
\end{gathered}
$$

The corresponding expressions for the one-dimensional case, as mentioned above, coincide with the threedimensional case given by Eqs. (A14-A16).

\section{Acknowledgements}

I am grateful to V. P. Krasin and S. I. Soyustova for their help in making plots. 


\section{References}

[8] E. A. Hylleraas, Z. Phys., 63, 291 (1930).

[1] A. Gorlitz et al., Phys. Rev. Lett., 87, 130402 (2001).

[9] E. A. Hylleraas, Z. Phys., 63, 771 (1930).

[2] U. Eichmann, V. Lange, and W. Sandner, Phys. Rev. Lett., 64, 274 (1990).

[3] V. V. Skobelev, JETP, 125, No. 6, 1058 (2017).

[4] V. V. Skobelev, JETP, 126, No. 2, 183 (2018).

[5] V. V. Skobelev, JETP, 126, No. 5, 645 (2018).

[6] A. A. Sokolov, Yu. M. Loskutov, and I. M. Ternov, Quantum Mechanics, Holt, Rinehart \& Winston, Austin, Texas (1966).

[7] L. D. Landau and E. M. Lifshitz, Theoretical Physics, Vol. 3, Quantum Mechanics: Non-Relativistic Theory, Third Edition, Pergamon Press, Oxford (1977).

[10] R. London, Amer. J. Phys., 27, 649 (1959).

[11] I. S. Gradshteyn and I. M. Ryzhik, Tables of Integrals, Series, and Products, Eighth Edition, Academic Press, Elsevier, Oxford (2014).

[12] V. V. Skobelev, JETP, 126, No. 3, 333 (2018).

[13] V. V. Skobelev, Russ. Phys. J., 58, No. 2, 163 (2015).

[14] B. Zaslow and C. E. Zandler, Amer. J. Phys., 35, 1118 (1967).

[15] A. Cisneros and N. V. McIntosh, J. Math. Phys., 10, 277 (1968). 